Public health

\section{Public health training in genitourinary medicine}

\section{A J Robinson, N Payne, H Ward}

GUM consultants need skills that fall into the broad area of public health

G enitourinary medicine (GUM) is a clinical specialty. In common with other specialties, clinical training alone is insufficient to prepare consultants for life in the modern NHS. Consultants need to have a wide range of skills from other disciplines if they are to provide and manage an effective service. In addition to generic skills needed for all NHS consultants, specialists in GUM need to understand the role of treatment and care services in the control of sexually transmitted infections (STI). The current network of clinics in the United Kingdom dates back to the Royal Commission report on venereal diseases of 1916 that recommended services be "skilled, free... and provided at the earliest possible moment" in order to reduce the impact of infection on the population-that is, these services were developed and maintained to deliver a public health goal. ${ }^{12}$ Thus the consultant in GUM has always had to address the need of individual patients attending services and the need of the population for infection control and health promotion. Over the past 5 years there has been a renewed emphasis on these aspects of GUM, with sexual health and HIV highlighted as a public health priority. ${ }^{3}$

Rapid change in the delivery of sexual health services in the United Kingdom is expanding the range of sexual health providers to include non-specialists, nurses, and other health professionals. In this context, the consultant specialists in GUM will need to provide leadership and vision in developing networks, shared care pathways, clinical governance, and training for key providers. They will also need to ensure that population health remains at the forefront of decisions on service configuration and local strategy. For consultants to take on this leadership role they need a number of skills that fall into the broad area of public health. It is not sufficient for a consultant to provide a treatment and care service for the patients who come through their open door. They should understand the epidemiology of HIV and STI in their local population, and have the appropriate skills to enhance control mechanisms and outbreak response. They need to understand the local health service, be able to assess population needs and the impact of change, and develop skills in partnership working. The entire justification for a free, low threshold, open access service is to contribute to the control of infection in the population, and GUM specialists must champion this to improve the sexual health of the nation.

Acquiring such skills is not automatic and needs to be systematised. At the moment the training of doctors is undergoing fundamental change. Trainees have been expected to undertake basic specialist training and achieved the necessary examinations before enrolling in a 4 year higher specialist training programme, during which the trainee acquires skills in other clinical and laboratory disciplines. At the moment, aspects of public health are covered at many points during this process, although not in a strictly defined way. With modernising medical careers, the "free range" approach to gaining broad experience across a range of specialties will no longer be viable. It is unclear exactly how training will follow foundation programmes but it seems likely that there will be a less flexible approach. Given the centrality of public health, the specialty has recently agreed to include some defined areas of public health training within the specialist training curriculum. This will help future consultants to gain skills to enable them to run GUM services in a changing healthcare system. The influence of the specialty at a strategic level will be increased to the benefit of the services and outcomes for individual patients as well as the public health.

The Faculty of Public Health in the United Kingdom has defined competencies and many of these are relevant (www.fphm.org.uk). The core competencies are the surveillance and assessment of the population's health and wellbeing; assessing the evidence of effectiveness of health and healthcare interventions, programmes, and services: policy and strategy development and implementation; and strategic leadership and collaborative working for health. In addition, specific competencies relevant to GUM are health improvement and health protection. Table 1 shows how these may be related to GUM. These public health competencies should be delivered throughout specialist training with theoretical, practical, and assessment components. Suggested models include the expansion of the BASHH/HPA fellowship programme, in which trainees spend one day a week for a year working with the Health Protection Agency at national or regional level; modular training using existing and new courses-for example, an additional module in the BASHH SpR course, the practical application of knowledge and skills-for example, through projects conducted jointly with local public health specialists, health protection unit, or academic department. To deliver this input, leadership from those consultants with dual specialist registration in GUM and public health is needed. And, in parallel, development of a network of public health specialists with an interest in sexual health and communicable disease control would facilitate this process.

Sex Transm Infect 2006;82:191-192.

doi: $10.1136 /$ sti.2006.021345

\section{Authors' affiliations}

A J Robinson, Department of Genitourinary Medicine, Mortimer Market Centre, Camden PCT, London WCIE 6AU, UK

N Payne, North Eastern Derbyshire Primary Care Trust, Chesterfield, S41 7TD, UK

H Ward, Department of Infectious Disease Epidemiology, Imperial College Faculty of Medicine, London W2 IPG, UK

Correspondence to: Dr Helen Ward, Department of Infectious Disease Epidemiology, Imperial College Faculty of Medicine, London W2 1PG, UK; h.ward@imperial.ac.uk

\section{REFERENCES}

1 Royal Commission on Venereal Diseases. Final report of the commissioners. London: HMSO 1916 (Cmnd 8189).

2 Evans D. Tackling the 'hideous scourge': the creation of the venereal disease treatment centres in early twentieth-century Britain. Social History of Medicine 1992;5:413-33.

3 Department of Health. The national strategy for sexual health and HIV. London: Department of Health, 2001:1-51.

4 Department of Health. Choosing health: making healthier choices easier. London: Department of Health, 2004. 


\section{Table 1}

\section{Competency}

Surveillance and assessment of the population's health and wellbeing This area of practice focuses on the assessment of the population's health, including managing, analysing, and interpreting information; knowledge and statistics related to the determinants and status of health and wellbeing; and the assessment of needs and outcomes related to health and wellbeing.

\section{Assessing the evidence of effectiveness of health and healthcare} interventions, programmes, and services.

This area of practice focuses on the critical assessment of the evidence relating to the effectiveness of health and healthcare interventions, programmes, and services; applying this to practice; and improving services and interventions through audit and evaluation.

Policy and strategy development and implementation.

This area of practice focuses on influencing the development of policies, implementing strategies to put the policies into effect, and assessing the impact of policies on health.

Strategic leadership and collaborative working for health.

This area of practice focuses on leading teams and individuals, building alliances, developing capacity and capability, working in partnership with other practitioners and agencies, and using effectively the media to improve health and wellbeing.

Health improvement.

This area of practice focuses on working with and for communities to improve health and reduce inequalities, and the application of health promotion theories to public health programmes, and theoretical models of behaviour change to promote health in the individual.

Health protection.

This area of practice focuses on applying knowledge of the nature, causes, and occurrence of major communicable and non-communicable diseases; and of the principles of the modes of transmission, latency, incubation periods, exposure, herd and individual immunity; and advising on different aspects of immunisation programmes to assessing, investigating, and communicating infectious and non-infectious environmental hazards and protecting the public against them.

\section{Examples relevant to genitourinary medicine}

A GUM consultant should have the skills required to assess the epidemiology of STI in their area. This would include describing the distribution of STI by classic epidemiological descriptors of time, person, and place.

The basic skills necessary would include an understanding of:

- Incidence and prevalence

- Surveillance methods: routine data and their interpretation

- Data sources

- Rates

- Standardisation

- Research methods including epidemiological study design, the appropriate use and interpretation of common statistical analyses, and the interpretation of research findings.

- Methods for summarising and presenting data and research findings.

Also required would be skills at presenting and communicating the findings to a variety of audiences and making recommendations for action.

This is often summarised as "clinical epidemiology." A GUM consultant should be competent in applying the basics of clinical epidemiology to the management of their own patients (in interpretation of findings and choice of management) and to the running of an effective service (including when to introduce new screening tests, treatments, etc).

A GUM consultant should be able to carry out a critical appraisal of the literature relevant to a clinical intervention such as a therapeutic procedure, diagnostic test, or prognostic assessment.

Included with this competency is an understanding of the principles of health economics - particularly as applied to the health technology appraisal and assessment process conducted by NICE and other similar organisations.

A GUM consultant should be able to understand how policy is made, how to influence it and how to make an appropriate case for investment and development of their service. This would include a knowledge of how the NHS and other key organisations (for example, local government) are organised and function.

A GUM consultant should have appropriate managerial and leadership skills as a clinical leader for their specialty

Relevant skills include:

- Understanding types of organisational structures

- Leadership style

- Change management

- Multiagency working

- Project management

- Use of media

Specific areas relevant to a GUM consultant are:

- Health promotion-understanding the relevant principles of health promotion in the context of sexual health. For example

- Effectiveness of interventions such as educational interventions for young people, condom provision, behaviour change interventions for high risk groups, needle exchange

- Screening-understanding key principles of screening-applied, for example, to chlamydia screening.

The GUM consultant should understand how the health protection function is organised in the UK so they can work closely with the local consultant in communicable disease control/health protection unit.

The consultant should have an understanding of the key components of HIV and STI control programmes and apply this to the development of their local servicesnamely,

- the key determinant of STI epidemics including the basic reproductive rate $R_{0}$

- principles and practice of health promotion and the role of clinicians in primary and secondary prevention

- the key role of timely access to diagnosis and treatment in STI control

- principles and practice of partner notification

- techniques for monitoring local trends and identifying outbreaks or clusters of disease

- principles and practice of management of an outbreak

- interventions available to control an outbreak and reduce STI in the local population. 